\title{
Aspectos reprodutivos de Goniopsis cruentata (Latreille) (Crustacea, Brachyura, Grapsidae) na Baía de Sepetiba, Rio de Janeiro, Brasil
}

\author{
Zilanda de Souza Silva ${ }^{1}$ \\ Lídia Miyako Yoshii Oshiro $^{1}$
}

\begin{abstract}
Reproductive aspects of Goniopsis cruentata (Latreille) (Crustacea, Brachyura, Grapsidae) at the Sepetiba Bay,Rio de Janeiro, Brazil. The objective of this study was to get some knowledge about the reproductive aspects of Goniopsis cruentata (Latreille, 1803), concerning through sex ratio, morphological sexual maturity, reproductive period, fecundity and eggs size. The crabs were collected monthly in Itacuruçá's Mangroves from November, 1997 to November, 1998. Four hundred and seven crabs were caught and they were $47 \%$ males, $36 \%$ females and $17 \%$ ovigerous females. The sex ratio was 1:1.13 (male:female) $\left(X^{2}=1.54, p>0.05\right)$. The relation of abdomen width (AW) and carapace width $(\mathrm{CW})$ in females showed a positive allometric grow $(b=1.36)$, it suggests the size onset of maturity occur from 26.0 a $29.0 \mathrm{~mm}(\mathrm{CW})$ in females. The ovigerous females occured during all the research period, except for the winter months (June to August). The size of these ovigerous females varied from 26.0 to $48.8 \mathrm{~mm}$ with mean size $35.1 \pm 5.2 \mathrm{~mm}$ Individual fecundity varied from 29,975 to 142,050 eggs and mean fecundity was $74,751 \pm 27$ eggs. The mean diameter of the eggs was $300 \pm 20 \mu \mathrm{m}$.

KEY WORDS. Grapsidae, Goniopsis cruentata, reproduction, fecundity, sexual maturity, Sepetiba Bay
\end{abstract}

No Brasil, Grapsidae apresenta quinze gêneros e vinte e duas espécies (MELO 1996). Segundo OsHIRO et al. (1998) nos Manguezais da Baía de Sepetiba essa família está representada por cinco espécie: Goniopsis cruentata (Latreille, 1803), espécie considerada muito abundante, Aratus pisonii (H. Milne Edwards, 1837), Chasmagnathus granulata Dana, 1851, Metasesarma rubripes (Rathbun, 1897) e Sesarma rectum Randall, 1840.

Goniopsis cruentata é um caranguejo semiterrestre conhecido popularmente como aratu ou mulata (MELo 1996) e é uma espécie de importância econômica na Região Nordeste do Brasil, utilizada como fonte de alimento e se distribui no Atlântico Ocidental - nas Bermudas, Flórida, Golfo do México, Antilhas, Guianas, Brasil (Fernando de Noronha e do Pará até Santa Catarina) - e no Atlântico Oriental do Senegal até Angola (MELO 1996).

Trabalhos realizados com essa espécie, ainda são escassos destacando-se, no Brasil, aqueles realizados por: FRANSOZO et al. (1998) que descreveram o estágio larval zoea I; COBO \& FrANSOZO (1998, 1999), que estudaram uma população em Ubatuba, São Paulo, sobre os aspectos de reprodução e crescimento. Na Baía de Sepetiba, os aspectos reprodutivos em braquiúros foram estudados apenas para as

1) Estação de Biologia Marinha, Universidade Federal Rural do Rio de Janeiro. Rua Sereder, 23860-000 Itacuruçá, Mangaratiba, Rio de Janeiro, Brasil. E-mail: oshiro@ufrrj.br

Revta bras. Zool. 19 (3): 907 - 914, 2002 
seguintes espécies: Callinectes danae Smith, 1869 (MEDEIROS \& OSHIRO 1992) e Menippe nodifrons Stimpson, 1859 (OSHIRO 1999).

O presente trabalho teve como objetivo obter dados sobre os aspectos reprodutivos de G. cruentata, no manguezal de Itacuruçá, na Baía de Sepetiba, Rio de Janeiro, especificamente no que se refere à razão sexual, maturidade sexual morfológica, período reprodutivo, fecundidade e tamanho dos ovos, visando contribuir para ampliar o conhecimento da bioecologia da espécie.

\section{MATERIAL E MÉTODOS}

O Manguezal de Itacuruçá localiza-se na Baía de Sepetiba, Município de Mangaratiba, Estado do Rio de Janeiro (22 $54^{\prime} 06^{\prime \prime}-23^{\circ} 02^{\prime} 30^{\prime \prime}$ S e $43^{\circ} 33^{\prime} 42^{\prime \prime}$ $44^{\circ} 02^{\prime} 30^{\prime \prime} \mathrm{W}$ ) e tem aproximadamente 10 ha de área.

Os caranguejos foram coletados mensalmente, de novembro de 1997 a novembro de 1998, sempre durante o dia e na maré baixa, com exceção do mês de abril/98, devido às más condições climáticas. As coletas foram efetuadas utilizando-se o esforço de dois coletores, durante o período de 90 minutos.

Os exemplares foram capturados com puçás e colocados em baldes, separados por sexo, de acordo com a morfologia do abdome, mensurados com o uso de um paquímetro $(0,05 \mathrm{~mm})$ e devolvidos ao ambiente.

Para a análise morfométrica foram realizadas as mensurações das seguintes dimensões corpóreas: largura da carapaça (LC); largura do abdome (quarto somito) (LA) e altura do própodo quelar (AP).

Para verificar a existência, ou não, de diferenças significativas na razão sexual mensal ao longo do ano, utilizou-se o teste do qui-quadrado $\left(X^{2}\right)$ ao nível de $5 \%$.

As relações morfométricas analisadas foram: LA x LC nas fêmeas e AP $\mathrm{x}$ LC nos machos. Essas relações foram analisadas através da análise de regressão e as equações que descreveram essas relações foram do tipo $\mathrm{y}=\mathrm{ax}^{\mathrm{b}}$, onde: $\mathrm{x}=$ variável independente (LC); $\mathrm{y}$ = variável dependente (LA e AP); onde b foi a constante de crescimento relativo.

De acordo com a morfologia do abdome, os animais foram divididos nos seguintes grupos: machos jovens, fêmeas jovens, machos adultos, fêmeas adultas (COBO \& FRANSOZO 1998).

Para verificar a existência, ou não, de diferenças no crescimento entre os grupos estudados (jovens e adultos, machos e fêmeas), utilizou-se o método de comparação do coeficiente de regressão b (constante de crescimento), através do Teste "t" de Student, a nível de 5\%.

O período reprodutivo foi determinado através da presença de fêmeas ovígeras ao longo do período de estudo.

As fêmeas ovígeras foram separadas e devolvidas ao ambiente após a retirada da massa de ovos dos pleópodos. A massa de ovos foi colocada em frasco e congelada. Posteriormente, os ovos foram dissociados com uma solução de hipoclorito de sódio a 100\%, lavados com água corrente e colocados em álcool a $70 \%$ para conservação. 
A fecundidade foi estimada através de uma amostragem de 51 fêmeas ovígeras, onde os ovos de cada fêmea, foram colocados num becker com $75 \mathrm{ml}$ de água. Foram retiradas três subamostras de $1 \mathrm{ml}$ cada, após a homogeneização, e contadas após serem distribuídas em uma câmara quadriculada e com auxílio de um contador manual, sob um estereomicroscópio binocular.

A relação entre a largura da carapaça $(\mathrm{LC})$ e a fecundidade $(\mathrm{F})$ foi expressa pela equação $\mathrm{F}=\mathrm{aLC}^{\mathrm{b}}$.

O diâmetro dos ovos foi mensurado aleatoriamente em 20 ovos de cada fêmea, utilizando-se estereomicroscópio com ocular de medição (aumento de 25x).

\section{RESULTADOS E DISCUSSÃO}

Dos 407 caranguejos capturados, $47 \%$ foram machos, $36 \%$ fêmeas e $17 \%$ fêmeas ovígeras. A razão sexual foi de 1:1,13 (macho:fêmea) $\left(X^{2}=1,54 ; p>0,05\right)$. Houve um pequeno predomínio de fêmeas sobre os machos em quase todo o período, com exceção de novembro/1997, fevereiro/1998, julho/1998, novembro/1998, não tendo sido verificada diferença significativa $(\mathrm{p}>0,05)$ entre os meses do período estudado (Tab. I).

Tabela I. Goniopsis cruentata. Freqüência absoluta de machos e fêmeas de indivíduos capturados de novembro/97 a novembro/98, razão sexual (machos:fêmeas) e teste do Quiquadrado, no Manguezal de Itacuruçá, Rio de Janeiro.

\begin{tabular}{lccccc}
\hline \multicolumn{1}{c}{ Meses } & Machos & Fêmeas & Machos/têmeas & Fêmeas ovigeras & $x^{2}\left({ }^{*}\right)$ \\
\hline Novembro/1997 & 18 & 17 & $1: 0,94$ & 5 & 0,028 \\
Dezembro & 16 & 18 & $1: 1,13$ & 10 & 0,059 \\
Janeiro/1998 & 16 & 19 & $1: 1,19$ & 10 & 0,257 \\
Fevereiro & 17 & 13 & $1: 0,76$ & 6 & 0,533 \\
Março & 13 & 17 & $1: 1,31$ & 9 & 0,533 \\
Maio $\left({ }^{*}\right)$ & 16 & 20 & $1: 1,25$ & 5 & 0,444 \\
Junho & 15 & 21 & $1: 1,40$ & - & 1,000 \\
Julho & 17 & 16 & $1: 0,94$ & - & 0,030 \\
Agosto & 17 & 20 & $1: 1,18$ & - & 0,243 \\
Setembro & 15 & 20 & $1: 1,33$ & 6 & 0,714 \\
Outubro & 13 & 18 & $1: 1,38$ & 9 & 0,806 \\
Novembro & 18 & 17 & $1: 0,94$ & 9 & 0,028 \\
\hline Total & 191 & 216 & $1: 1,13$ & 69 & 1,535 \\
\hline
\end{tabular}

${ }^{(*)}$ Em todos os casos não foi verificada diferença significativa na proporção macho:fêmea (p $>0,05) ;\left(^{* *}\right)$ não foi realizada coleta no mês de abril, devido a chuva nos horários de maré baixa durante o mês.

O tamanho nos machos variou de 21,5 a $56,0 \mathrm{~mm}$ de largura da carapaça, apresentando o tamanho médio de $36,0 \pm 7,3 \mathrm{~mm}$. As fêmeas não ovígeras variaram de 22,2 a $48,8 \mathrm{~mm}$, com tamanho médio de $35,1 \pm 5,21 \mathrm{~mm}$ e as fêmeas ovígeras variando de 26,0 a 48,8 $\mathrm{mm}$ com tamanho médio de 37,8 $\pm 4,1 \mathrm{~mm}$ (Figs 1 e 2). COBO \& FRANSOZO (1999) encontraram numa população em Ubatuba, São Paulo, fêmeas ovígeras variando de $25,1 \mathrm{~mm}$ a $41,2 \mathrm{~mm}$.

A maturidade sexual morfológica em fêmeas foi estimada para o tamanho de 26,0 a $29,0 \mathrm{~mm}$ de largura da carapaça, onde verificou-se uma sobreposição no crescimento entre a largura da carapaça e o abdome, de jovens e adultas, indicando 


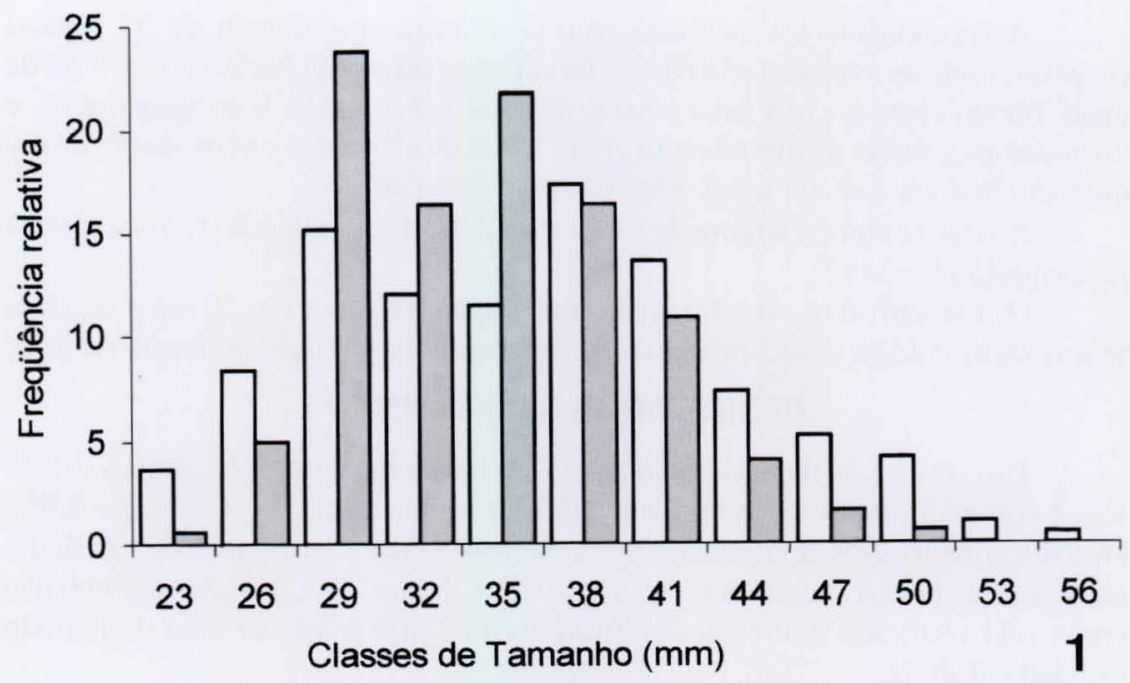

$\square$ Machos $N=191$ afêmeas $N=216$

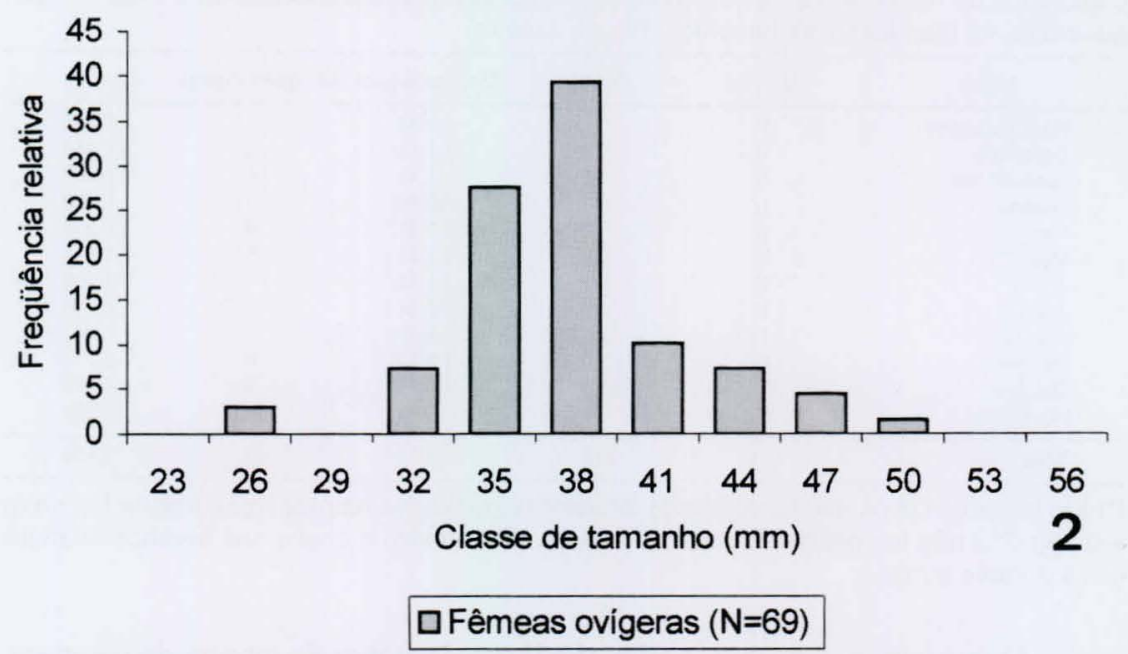

Figs 1-2. Goniopsis cruentata, distribuição da freqüência relativa de (1) machos e fêmeas e de (2) fêmeas ovígeras em classes de tamanho, no período de novembro/1997 a novembro/1998, no Manguezal de Itacuruçá, Rio de Janeiro.

a ocorrência da muda da puberdade nessa faixa de tamanho (Fig. 3). A equação que descreveu o crescimento de todas as fêmeas foi $\mathrm{LA}=0,173 \mathrm{LC}{ }^{1,36}\left(\mathrm{R}^{2}=0,89\right)$, enquanto das jovens foi $\mathrm{LA}=0,107 \mathrm{LC} \mathrm{C}^{1,47}\left(\mathrm{R}^{2}=0,94\right)$ e das adultas foi $\mathrm{LA}=$ $0,244 \mathrm{LC}^{1,26}\left(\mathrm{R}^{2}=0,87\right)$. 


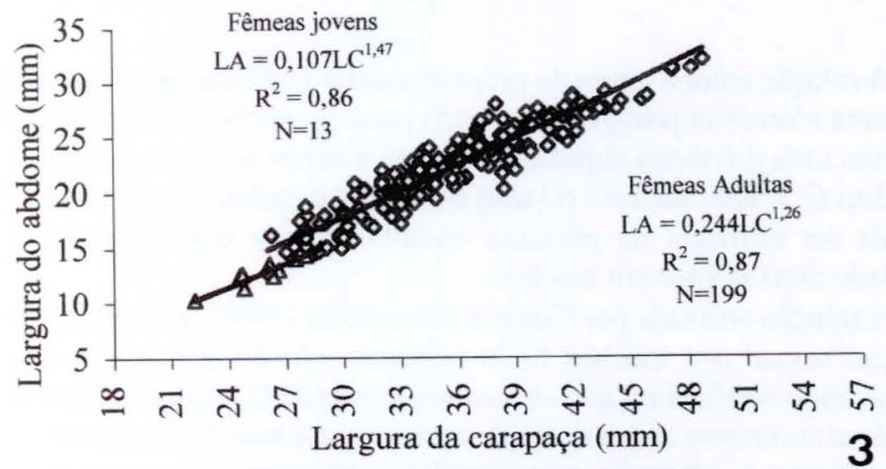

$\Delta$ Fêmeas jovens $\diamond$ Fêmeas adultas
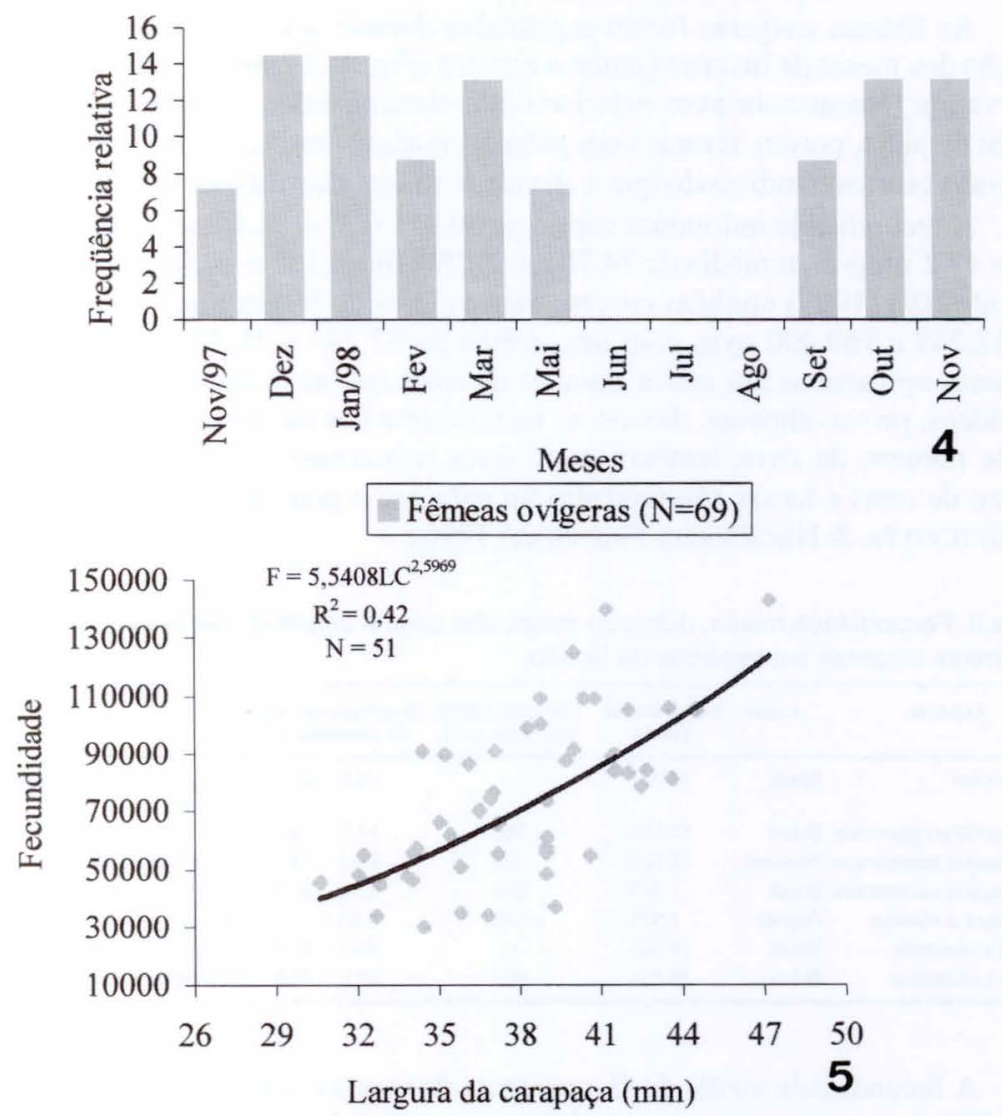

Figs 3-5. Goniopsis cruentata, no período de novembro/1997 a novembro/1998, no Manguezal de Itacuruçá, Rio de Janeiro. (3) Relação morfométrica entre a largura do abdome (LA) e a largura da carapaça (LC) para fêmeas jovens e fêmeas adultas; (4) distribuição mensal de fêmeas ovígeras; (5) relação entre a fecundidade $(F)$ a largura da carapaça $(L C)$ em fêmeas ovígeras. 
A relação entre a altura do própodo quelar (AP) e a largura da carapaça (LC) sugere uma alometria positiva $(b=1,65)$ para os machos, porém essa relação não apresentou uma diferença significativa entre machos jovens e machos adultos ( $\mathrm{p}>$ 0,001). Em G. cruentata, essa relação não segue o padrão dos braquiúros. Portanto, não pôde ser utilizada no presente trabalho para a determinação do início da maturidade morfológica em machos.

A relação utilizada por COBO \& FRANSOZO (1998), para verificar o início da maturação sexual nos machos foi o comprimento do gonopódio e a largura da carapaça, onde verificaram que os machos jovens de $G$. cruentata apresentaram um padrão de crescimento abdominal que acompanha a transformação dos gonopódios, pois o abdome é adaptado para guardar e proteger os gonopódios, apêndices relacionados diretamente à reprodução.

As fêmeas ovígeras foram registradas durante quase todo o período, com exceção dos meses de inverno (junho a agosto) (Fig. 4). COBO \& FRANSOZO (1999) observaram fêmeas com ovos exteriorizados durante todos os meses com exceção do mês de julho, porém, fêmeas com gônadas maduras estiveram presentes em todo o período estudado indicando que a atividade sexual dessa espécie é contínua.

A fecundidade individual variou de 29.975 ( $\mathrm{LC}=34,4 \mathrm{~mm}$ ) a 142.050 ovos ( $\mathrm{LC}=47,2 \mathrm{~mm}$ ) com média de $74.751 \pm 27.296$ ovos $(\mathrm{LC}=38,0 \pm 3,6 \mathrm{~mm})$. COBO \& FRANSOZO (1999) também encontraram valores de fecundidade bastante variáveis 12.249 a 169.400 ovos com uma média de $57.235 \pm 35.235$ ovos. Goniopsis cruentata apresentou um maior número de ovos quando comparados com outros grapsídeos, provavelmente, devido ao menor porte das outras espécies (Tab. II). O grande número de ovos também pode estar relacionado ao fato de que grande número de ovos e larvas são predadas na natureza e poucas larvas atingem a fase juvenil (COSTA \& NEGREIROS-FRANSOZO 1996).

Tabela II. Fecundidade média, diâmetro médio dos ovos e amplitude da largura da carapaça das fêmeas ovígeras em espécies da família.

\begin{tabular}{|c|c|c|c|c|c|}
\hline Espécie & Local & $\begin{array}{l}\text { Fecundidade } \\
\text { média }\end{array}$ & $\begin{array}{l}\text { Diâmetro médio } \\
\text { dos ovos }(\mu \mathrm{m})\end{array}$ & $\begin{array}{l}\text { Amplitude da largura } \\
\text { da carapaça }(\mathrm{mm})\end{array}$ & Autor \\
\hline Aratus pisonii & Brasil & 15.197 & - & $15,0-24,3$ & $\begin{array}{l}\text { Leme \& Negreiros-Fransozo } \\
\text { (1998) }\end{array}$ \\
\hline Chasmagnathus granulata & Brasii & 19.250 & 360 & $14,5-25,6$ & Ruffino et al. (1994) \\
\hline Pachygrapsus transversus & Panamá & 15.000 & 220 & $9,40-13,8$ & Abele et al. (1986) \\
\hline Pachygrapsus transversus & Brasil & 1.770 & 280 & $8,50-20,0$ & Campos \& Oshiro (2001) \\
\hline Pachygrapsus maurus & Açores & 1.771 & 240 & $5,20-11,6$ & Lmaça (1987) \\
\hline Goniopsis cruentata & Brasil & 57.235 & - & $25,1-41,4$ & Cobo \& Franzoso (1999) \\
\hline Goniopsis cruentata & Brasil & 74.751 & 300 & $26,0-48,8$ & Presente estudo \\
\hline
\end{tabular}

A fecundidade média de $G$. cruentata de acordo com as classes de tamanho (Tab. III), demonstrou que essa espécie segue o padrão dos crustáceos braquiúros com o número de ovos aumentando com o tamanho das fêmeas. Este padrão foi observado por COSTA \& NEGREIROS-FRANSOZO (1996) em Callinectes danae Smith, 1869; MantelatTo \& Fransozo (1997) em Callinectes ornatus Ordway, 1863 e LEME \& NegreIros-Fransozo (1998) em Aratus pisonii. 
Tabela III. Goniopsis cruentata, no Manguezal de Itacuruçá, Rio de Janeiro. Fecundidade média por classe de tamanho em fêmeas ovígeras.

\begin{tabular}{cccc}
\hline Classe de tamanho $(\mathrm{mm})$ & $\mathrm{N}$ & Fecundidade média & Desvio padrão \\
\hline $30,5+33,5$ & 5 & 45.545 & 7.586 \\
$33,5+36,5$ & 12 & 59.810 & 20.285 \\
$36,5+39,5$ & 16 & 69.169 & 22.031 \\
$39,5+42,5$ & 12 & 97.062 & 21.931 \\
$42,5+45,5$ & 5 & 90.675 & 13.356 \\
$45,5+48,5$ & 1 & 142.050 & - \\
\hline
\end{tabular}

A relação da fecundidade e largura da carapaça, no presente trabalho pode ser descrita pela equação $F=5,541 L_{C}^{2,597}\left(R^{2}=0,42\right)$ (Fig. 5), enquanto CoBo \& FRANSOZO (1999) descreveram esta mesma relação pela equação y $=0,084 x^{3,8}\left(r^{2}\right.$ $=0,61$ ). O baixo coeficiente de determinação, indica que apenas uma parte da variação da fecundidade pode ser atribuída ao tamanho do animal.

Segundo HiNES (1982) e HARTNOLL (1969), os valores contrastantes do número de ovos em animais de tamanhos semelhantes, podem ser decorrentes de desovas múltiplas ou pelo fato das fêmeas perderem ovos durante a incubação, por ataque de parasitas ou pela atividade mecânica dos caranguejos atritando contra o substrato.

Segundo COSTA \& NEGREIROS-FRANSOzO (1996) são fatores que contribuem para a variação da fecundidade: a estação do ano; a disponibilidade de alimento no ambiente e a fase do período reprodutivo de cada fêmea (número de desovas do ciclo).

O tamanho médio dos ovos foi de $300 \pm 20 \mu \mathrm{m}$ variando de 240 a $360 \mu \mathrm{m}$. Comparando o tamanho dos ovos de G. cruentata com o de outros grapsídeos (Tab. II), verifica-se seu menor tamanho. Segundo NEGREIROS-FRANSOZO et al. (1992), o tamanho dos ovos tem importante consequiência na razão de desenvolvimento e no tamanho das larvas e juvenis, ovos maiores produzem descendentes melhor adaptados para alimentação e com maiores habilidades competitivas.

AGRADECIMENTO. Ao Senhor Casemiro Antonio Alves, pelo auxílio nas coletas.

\section{REFERÊNCIAS BIBLIOGRÁFICAS}

Abele, L.G.; P.J. Campanela \& M. Salmon. 1986. Natural history and social organization of the semiterrestrial grapsid crab Pachygrapsus transversus (Gibbes). Jour. Exp. Mar. Biol. Ecol., Amsterdam, 104: 153-170.

AlmaÇA, C. 1987. Egg number and size in Pachygrapsus maurus (Lucas, 1846) from Praia da Laginha (Faial, Azores islands). Inv. Pesq., Santiago, 51 (10): 157-163.

CAMPoS, D.A. \& L.M.Y. OshiRO. 2001. Biologia reprodutiva do caranguejo Pachygrapsus transversus (Gibbes, 1850) (Crustacea, Decapoda, Grapsidae) da Praia de Ibicuí-RJ. X Jornada Científica da UFRRJ, Trabalhos Completos, 11 (2): 209-212.

Сobo, V. J. \& A. Fransozo. 1998. Relative growth of Goniopsis cruentata (Crustacea, Brachyura, Grapsidae), on the Ubatuba Region, São Paulo, Brazil. Iheringia, Sér. Zool., Porto Alegre, (84): 21-28. 
1999. Fecundity and reprodution period of the red mangrove crab Goniopsis cruentata (Brachyura, Grapsidae) São Paulo state, Brazil, p. 527-533. In.: J.C. von VAupel KLeIn \& F.R. SCHRAM (Eds). The Biodiversity Crisis and Crustacea. Amsterdam, A.A. Balkema Publishers, Crustacean Issues, Vol. 12, 800p.

Costa, T.M. \& M.L. Negreiros-Fransozo. 1996. Fecundidade de Callinectes danae Smith, 1869 (Crustacea, Decapoda, Portunidae) na Região de Ubatuba (SP), Brasil. Arq. Biol. Tecnol., Curitiba, 39 (2): $393-400$.

Fransozo, A.; J.A. Cuesta \& M.L. Negreiros-Fransozo. 1998. The first zoeal stage of two species of Grapsidae (Decapoda, Brachyura) and a key to such larvae from the brazilian coast. Crustaceana, Netherlands, 71 (3): 331-343.

HaRTNolL, R.G. 1969. Mating in the brachyura. Crustaceana, Netherlands, 16: 161-181.

HiNES, A.H. 1982. Allometric constraints and variables of reproductive effort in brachyuran crabs. Mar. Biol., Heidelberg, 69: 309-320.

Leme, M.H.A. \& M.L. Negreiros-Fransozo. 1998. Fecundity Aratus pisonii (Decapoda, Grapsidae) in Ubatuba Region, State of São Paulo, Brazil. Iheringia, Sér. Zool., Porto Alegre, (84): 73-77.

Mantelatto, F.L.M. \& A. Fransozo. 1997. Fecundity of the crab Callinectes ornatus Ordway, 1863 (Decapoda, Brachyura, Portunidae) from the Ubatuba Region, São Paulo, Brazil. Crustaceana, Netherlands, 70 (2): 214-226.

MEdeiros, M.F.S.T. \& L.M.Y. Oshiro. 1992. Aspectos reprodutivos de Callinectes danae Smith, 1869 (Crustacea, Decapoda, Portunidae), na Baía de Sepetiba - RJ. In: II Simpósio de Ecossistemas da costa sul e sudeste Brasileira, estrutura, função e manejo. ACIESP 4, Águas de Lindóia, 71: 150-159.

MELo, G.A.S. 1996. Manual de identificação dos Brachyura (caranguejos e siris) do litoral brasileiro. São Paulo, Ed. Plêiade, 603p.

Negreiros-Fransozo, M.L.; A. Fransozo; F.L.M. Mantelatto; J.M. Nakagaki \& M.C.F. SPILBORGHS. 1992. Fecundity of Paguristes tortugae Schmitt, 1933 (Crustacea, Decapoda, Anomura) in Ubatuba (SP) Brazil. Rev. Brasil. Biol., Rio de Janeiro, 52 (4): 547-553.

Oshiro, L.M. O. 1999. Aspectos reprodutivos do caranguejo guaia, Menippe nodifrons Stimpson (Crustacea, Decapoda, Xanthidae) da Baía de Sepetiba, Rio de Janeiro, Brasil. Revta bras. Zool., Curitiba, 16 (3):827-834.

Oshiro, L.M.O.; R. Silva \& Z.S. Silva. 1998. Composição da fauna de braquiúros (Crustacea, Decapoda) dos Manguezais da Baía de Sepetiba - RJ. Nauplius, Rio Grande, 6: 31-40.

RufFino, M.L.; M.D. Telles \& F. D'InCAO. 1994. Reproductive aspects of Chasmagnathus granulata Dana, 1851 (Decapoda, Grapsidae) in the Patos Lagoon Estuary - Brazil. Nauplius, Rio Grande, 2: $43-52$.

Recebido em 20.XII.2001; aceito em 04.IX.2002. 